\title{
Application of Intelligent Garment Formwork Technology on Modern Garment Enterprises
}

\author{
Xiaobing Yang ${ }^{1}$ \\ ${ }^{1}$ Jilin Engineering Normal University, Changchun, Jilin, 135000
}

Keywords: intelligent garment formwork; modern garment enterprise; application research

\begin{abstract}
In response to the current intensified competition in the garment industry and the increasingly short product life cycle, in order to increase the response speed of the apparel industry, it is necessary to research on garment manufacturing process control from the perspective of industrial engineering (IE) and application of garment formwork technology to ensure the effective implementation of apparel IE management. Through the process analysis, production line optimization and standardized operation set the beat, balance the production line and other aspects of the study of the garment manufacturing process, and design and develop intelligent garment process templates, to improve the efficiency of more than $48 \%$, so that the garment process design, production, and the operation are all intelligent, boosting the full use of apparel CIMS in garment factories, and promoting the realization of intelligent, digital, and networking goals in the apparel manufacturing process.
\end{abstract}

\section{Introduction}

In the 1960s, Germany had begun to use stencil technology in the production of shirts. At the time, the sleeves, collars, and other parts of the shirts used templates. The material used for the stencil production was steel plates. After the 1980s, in Taiwan, China began to use plexiglass to make garment templates. The biggest problem with the template at that time was that the organic glass was not easy to preserve. With the advancement of science and technology, PVC plates, acrylic plates and other materials are gradually used in clothing templates. 90 years after the 20th century, China, Guangdong, Fujian and other places gradually began to use clothing templates, but only used locally, the main use of parts include collars, pockets and sleeves. Into the 21st century, due to the rising costs of labor, raw materials, etc., the demand for automation, digitalization, and intelligent production of apparel companies has increased, and the thinking and research and development of garment sewing machine manufacturers' automated production of garment sewing have also been triggered. With the rise of the long arm sewing machine, the clothing formwork technology is no longer limited to a certain part, but the entire piece of clothing can be used as a clothing template.

\section{Clothing Smart Manufacturing Process}

Industrial engineering is the discipline that designs, improves, and implements integrated manufacturing systems for people, materials, and equipment. Clothing IE (Industrial Engineering) is the use of various lean tools to plan, design, evaluate, and innovate garment production lines. It is systematic, comprehensive, and constantly optimized. Through job research, process optimization, process scheduling, production scheduling, etc. Means to reduce on-site waste and solve problems in the manufacturing process. Clothing IE combined with apparel template technology can greatly enhance the implementation of IE promotion. Industrial engineering will focus on solving the problem of garment production lines.

Modern garment industry production lines are mostly produced in an assembly line. How to divide and manage processes is an important part of an organization's production line. First of all, we must formulate the process rules, then carry out job research and action analysis for each operation unit, calculate the cycle of the assembly line, optimize the process, and ensure that the 
assembly line can run smoothly [4]. To make the production line run efficiently, process optimization and management must be increased for the bottleneck process, which can be assisted by intelligent process analysis software. Through the simplification, merger, improvement, omission and other methods to improve and optimize the processing process. Scientifically and rationally choose the process of applying intelligent garment process templates to increase production efficiency.

Standardization of operations is an important means for companies to improve the level of production line management. The so-called job standardization is the unified analysis of the entire operating system, analysis, research, and improvement of each process, process, and operation to gradually achieve efficient, accurate, and labor-saving operation results, and to form improved processes and actions. The operating standards are implemented in production. Traditional garment processing improves the production efficiency through the development of various fixtures and molds to ensure product quality. The intelligent garment craft template is an upgraded version of the combined mould, which enables the operational standardization to be effectively implemented.

Process synchronization and production balance are important signs of smooth production lines. The difficulty of process formulation is too great or too small, and the operator's skill level or skill level can cause disjointed production or congestion and affect the working efficiency of the production line. The application of the intelligent garment process template can reduce the difficulty of the operation and enable each process of the production line to be close to the multiple of the production beat or rhythm to achieve the continuity, balance, high efficiency, production scheduling and management of the production line.

To develop a garment company, it is necessary to have a correct scientific decision, but it must also have a strong enforcement. In field management, execution power is reflected in the ability to complete each operating unit accurately, quickly, and efficiently. The application of the intelligent clothing craft template effectively guarantees the execution of the garment processing link. The intelligent garment process template stylizes standardized operations, and employees can complete the processing tasks according to standard job actions. The intelligent clothing craft template is the ruler of the clothing operation specification action, it can guarantee the operation is accurate and fast. Combining timely clothing production (JIT) or smart hanging line (FMS) with garment formwork technology is conducive to the realization of IE management goals and the goal of improving production efficiency.

\section{Intelligent Clothing Craft Template Development and Application}

From the perspective of industrial engineering, the development of intelligent garment process templates is based on process analysis. First, the process task scope for applying garment process templates is determined. The cycle of assembly lines is used as the starting point, and the IE method is used in conjunction with garment CAPP (computer-aided process design). Optimize and simplify the process, and finally standardize the operation of the group. According to the operation standard book design template, the template of the garment template CAD is used to draw the template structure diagram, and the intelligent garment craft template of the set of processes is generated after being cut through the automated cutting equipment. The IE method [7] was used to analyze the new process after the application of the template, the balance analysis of the production line, the production line efficiency study, the job specification study, and the process flow optimization analysis. After the template was improved, it was promoted in similar enterprises.

Ordinary garment process template, design and development with manual drawings, hand-made completed, push forward when applied. Intelligent clothing process template, CAD template drawing and sewing path files are used when designing, intelligent template cutting equipment is used to complete the production, and automatic sewing machine is used for sewing [8] to realize the design, production and operation of garment process template. Automation and intelligence. After the garment craft template is developed, the developer puts the video, instructions and precautions of the operating procedure on the network, and creates a special two-dimensional code for the garment craft template, which is attached to the garment craft template. After the production line 
employee gets the garment craft template, scanning the QR code can see the usage and requirements of the garment craft template. Or create an electronic tag, get the operation of the process, RFID radio frequency scanning, and the automatic die casting machine directly runs the specified program and runs according to the set trajectory. In actual operation, the IoT devices such as sensors and wireless sensor networks are connected to the workshop monitoring server to dynamically collect various types of workshop information, analyze related data when necessary, and propose improvement suggestions to the management. Managers can exchange information with each workstation through the MES system to control and control the workshop in a timely manner to realize intelligent identification, positioning, tracking, monitoring and management of the production process.

\section{Application Effect Evaluation}

Through a large number of actual tests in Hebei Shangyu Garment Co., Ltd., we obtained: $101 \mathrm{~s}$ for manual bag opening, $48 \mathrm{~s}$ for application template opening, efficiency increased by $110 \%, 326 \mathrm{~s}$ for manual splicing, and 220 when applied stencils s, the efficiency is increased by $48 \%$; The time for manual opening of the bag is $315 \mathrm{~s}$, the time for the template to open the bag is $80 \mathrm{~s}$, and the efficiency is increased by $294 \%$. It can be seen from this that the efficiency has increased by $48 \%$ at least, and the maximum by $294 \%$. This has reduced the number of working hours per unit and improved the utilization rate of the working hours and production efficiency [9]. At the same time, due to the consolidation of some processes, the number of processes has been reduced and labor costs have been saved. The intelligent garment process template has unique advantages in sewing patterns, irregular patterns, quilting, etc. The intelligent transporter can realize printing sewing. The sewing effect is good and the quality is stable. Applying garment technology templates, different operators of garment companies have different feelings. Through interviews with practitioners in different positions in various companies. The design and processing of intelligent garment process templates require labor and materials, and this adds a certain cost. Therefore, the intelligent garment process templates are more suitable for use in uniforms, tooling, and foreign trade apparel production lines with large batches, high quality requirements, and high added value. Developers of smart clothing process templates need specialized knowledge in clothing samples, processes, CAD, etc., as well as IE project management knowledge such as process scheduling and production line balancing. When the production line is promoted, various production-related departments need to cooperate and update the management methods and concepts to ensure the smooth completion of the production line transformation.

The use of intelligent equipment and apparel template technology solves the complex process simplification problem, completely reduces the requirements for artificial technology, reduces the supporting staff on the production line, reduces the company's dependence on all-round employees, and solves the recruitment of the garment industry. Difficulties, especially those that are more difficult to find, reduce the costs of training employees, and to a certain extent, reduce the loss of employees.

The garment template has changed the mode of production. At the same time, it has created favorable conditions for the standardization of management. Enterprises can directly use the MES and ERP information technology to control the production process, and establish a unified production technology standard document according to the template technology. Managers can exchange information with various workstations through the information system to control and control the workshop in a timely manner to realize intelligent identification, positioning, tracking, monitoring and management of the production process. The application of garment formwork technology explains the value concept of textile and garment companies. The production method of assembly line will become the leading factor in the intelligent era. The garment formwork will also continue to improve and develop. It will promote the subversive transformation of the production pattern of the entire textile and garment enterprises and will accelerate the textile industry. The pace of transformation and upgrading of the garment industry. The development and application of intelligent equipment and clothing templates have completely changed the traditional operating 
methods of garment companies, maximizing production efficiency and saving a lot of time. The production process was balanced, the assembly line was made smoother, the production schedule was accurately grasped, and the production method on a just-in-time basis was realized.

\section{Conclusion}

The production of garment formwork can ensure the consistency of garment specifications, improve the quality of garments, compensate for the errors in the production process caused by inexperienced processing personnel, fatigue, etc., as well as the reliance on high-end professional equipment, thus improving garment processing enterprises. The efficiency of work and products is one of the new modes of production of clothing.

\section{Acknowledgements}

Project Name (Level): Application Research of Intelligent Garment Formwork Technology in Modern Garment Enterprises (Provincial Level).

Project Number: JJKH2017182SK

\section{References}

[1] Cai Hong. Men's trousers double insert bag design and application of research [J]. Wool Spin Technology, 2015, 43 (9): 66 - 67.

[2] Li Peng, Liu Sha. Digital Factory helps smart manufacturing [J]. China Industry Review, 2015 (4): 43 - 47.

[3] Yang Chuan, Zhang Xuui, Qing Jun, et al. Template technology in the garment industry in the application and development [J]. Textile Herald, 2014 (9): 71 - 73.

[4] Zhang Zhongqi, male suit rapid response process system design and research [J]. Wool Spin Technology, 2014, 42 (9): 34 - 39.

[5] Shen Xiaolai, Hu Guangxiang. Application of IE to improve the efficiency of clothing production line [J]. Shanghai Textile Science and Technology, 2010 (11): 11 - 13.

[6] Mao Pengli, Wang Lijun, Fan Linchao. Sewing process parameters on the sewing of silver fiber sewing thread [J]. Wool Spin Technology, 2015, 43 (6): 65 - 67. 